\title{
Licófitas e samambaias da Serra do Itauajuri, município de Monte Alegre, Pará, Brasil
} Lycophytes and ferns of Serra do Itauajuri, municipality of Monte Alegre, Pará, Brazil

\author{
Julieta Pallos ${ }^{1,5}$, Luiz Armando de Araújo Góes-Neto ${ }^{2}$, Jeferson Miranda Costa ${ }^{3}$, Filipe Soares de Souza ${ }^{2}$ \\ \& Marcio Roberto Pietrobom ${ }^{4}$
}

\begin{abstract}
Resumo
Neste estudo são apresentadas as licófitas e samambaias inventariadas na Serra do Itauajuri, estado do Pará. Foram registradas 64 espécies distribuídas em 36 gêneros e 21 famílias, sendo uma família, um gênero e quatro espécies pertencentes à linhagem das licófitas e 20 famílias, 35 gêneros e 60 espécies à linhagem das samambaias. As famílias mais representativas foram Pteridaceae (19 spp.), Polypodiaceae (8) e Selaginellaceae (4). Os gêneros com maior riqueza específica foram Adiantum (9 spp.) e Selaginella (4). A maioria das espécies foi coletada como terrestre (35 spp., ca. 55\%), ocorrendo em matas ripárias (51 spp., ca. 80\%), com padrão de distribuição americana (46 spp., ca. 72\%). São apresentados dois novos registros para a Região Norte do Brasil (Asplenium pumilum e Marsilea ancylopoda) e dois para o estado do Pará (Ophioglossum nudicaule e Selaginella minima). A área estudada possui características ambientais bastante distintas do que comumente se observa na Amazônia brasileira, sendo uma área biologicamente importante. Os resultados obtidos neste trabalho reúnem informações que podem subsidiar a criação e a implementação da Unidade de Conservação da Serra do Itauajuri, o que garantirá a preservação de suas riquezas históricas e naturais.
\end{abstract}

Palavras-chave: Amazônia brasileira, florística, formações campestres, mata ripária, pteridófitas.

\begin{abstract}
In this study we present the lycophytes and ferns inventoried in the Serra do Itauajuri, Pará state. Sixty four species distributed in 36 genera and 21 families were registered, with one family, one genus, and four species belonging to the lycophytes lineage, and 20 families, 35 genera, and 60 species belonging to the ferns lineage. The most representative families were Pteridaceae (19 spp.), Polypodiaceae (8), and Selaginellaceae (4). The genera with highest species richness were Adiantum (9 spp.) and Selaginella (4). Most species were collected as terrestrial (35 spp., ca. 55\%), occurring in riparian forests (51 spp., ca. 80\%), with American distribution pattern (46 spp., ca. 72\%). We present two new records for the Northern Region of Brazil (Asplenium pumilum and Marsilea ancylopoda) and two for Pará state (Ophioglossum nudicaule and Selaginella minima). The studied area has distinct environmental characteristics than those commonly observed in Brazilian Amazonia, being a biologically important area. This results gathered information that can support the creation and implementation of the Serra do Itauajuri Conservation Unit, which will guarantee the preservation of its historic and natural wealth. Key words: brazilian Amazonia, floristics, grassland vegetation, riparian forest, pteridophytes.
\end{abstract}

\section{Introdução}

A maior riqueza de licófitas e samambaias é, sem dúvida, encontrada nas florestas tropicais úmidas e regiões sub-tropicais (Tryon \& Tryon 1982), sendo estes vegetais um componente importante da flora tropical (Ricci 1996). A Floresta Amazônica é a maior floresta tropical do planeta, com aproximadamente 4,2 milhões de $\mathrm{km}^{2}$ inseridos em território brasileiro, o que representa $49 \%$ de todo o Brasil (Brasil 2008).

\footnotetext{
'Museu Paraense Emílio Goeldi, Coordenação de Botânica, Av. Perimetral 1901, 66077-830, Belém, PA, Brasil.

${ }^{2}$ Universidade Federal de Minas Gerais, Inst. Ciências Biológicas, Av. Antônio Carlos 6627, 31270-901, Pampulha, Belo Horizonte, MG, Brasil.

${ }^{3}$ Instituto Federal de Educação, Ciência e Tecnologia do Pará, Campus Abaetetuba, R. Rio Grande do Sul 3322, 68440-000, Francilândia, Abaetetuba, PA, Brasil.

${ }^{4}$ Universidade Federal do Pará, Campus de Bragança, Inst. Estudos Costeiros, Al. Leandro Ribeiro s.n., 68370-000, Aldeia, Bragança, PA, Brasil.

${ }^{5}$ Autor para correspondência: jujupallos@hotmail.com
} 
Embora caracterizada por um endemismo muito baixo (Tryon \& Conant 1975), a flora de plantas vasculares sem sementes na Amazônia brasileira é estimada em 550 espécies (Prado \& Moran 2009), o que representa cerca de $44 \%$ do total de espécies registradas no país (Prado et al. 2015).

Com uma área de $1.248 .000 \mathrm{~km}^{2}$, o estado do Pará destaca-se pelas cerca de 350 espécies de licófitas e samambaias registradas em seu território (Silva \& Rosário 2008; Góes-Neto \& Pietrobom 2012; Góes-Neto et al. 2015). No entanto, apesar dos avanços obtidos no conhecimento da composição florística destes grupos vegetais no estado (Costa \& Pietrobom 2010), o Pará ainda possui diversas áreas pouco ou nada amostradas, o que representa uma lacuna nas informações sobre a pteridoflora local, dificultando o entendimento da biogeografia e ecologia destes vegetais (GóesNeto \& Pietrobom 2012). As áreas com o menor esforço de coletas no estado são as mesorregiões do Sudoeste, Sudeste e Baixo Amazonas, que em conjunto, compõem um vasto território, fazendo fronteira com outros estados brasileiros e com países que possuem expressiva riqueza específica das suas floras de licófitas e samambaias.

O município de Monte Alegre, localizado às margens do Rio Amazonas na mesorregião do Baixo Amazonas, está inserido na região que abriga o maior dentre os oito centros de endemismo amazônicos, o Centro de Endemismo Guiana (Silva et al. 2005; Avila-Pires et al. 2010). Além disso, Monte Alegre apresenta um grande potencial turístico com destaque para o grande número de cachoeiras, igarapés, savanas e riquezas herdadas dos povos antepassados, especialmente os sítios arqueológicos que possuem arte rupestre, motivo pelo qual cerca de $50 \%$ do seu território haver sido transformado em área protegida (Pará 2013). Ainda segundo esta fonte, outro atrativo do município são as serras que formam mirantes naturais, conjugando beleza cênica com riqueza biológica, aspectos importantes para serem utilizados como estratégias na preservação ambiental, bem como no desenvolvimento sócio-econômico da região.

A Serra do Itauajuri é uma das mais expressivas feições topográficas de Monte Alegre (Pará 2013). Sua vegetação é bastante diversificada, apresentando desde formações florestais, como matas de galeria e cerradões, até tipos campestres com afloramentos rochosos, além disso, apresenta inúmeras cachoeiras e piscinas naturais (Pará 2013). O bom estado de conservação em que se encontra grande parte da vegetação local, bem como a significativa diversidade de ambientes com fisionomias pouco comuns ao bioma amazônico, proporcionam condições propícias ao estabelecimento de uma rica flora de licófitas e samambaias.

Assim, como muitas espécies desses grupos vegetais são consideradas importantes indicadores biológicos, utilizados no planejamento da conservação da biodiversidade na Amazônia (Zuquim et al. 2008), este estudo objetivou inventariar as espécies de licófitas e samambaias da Serra do Itauajuri, no município de Monte Alegre (PA). Além disso, são apresentadas informações sobre formas de vida, ambientes de ocorrência e padrões de distribuição geográfica dos táxons registrados, com o intuito de incrementar o conhecimento da pteridoflora amazônica e fornecer subsídios para o processo de criação da Unidade de Conservação da Serra do Itauajuri.

\section{Material e Métodos}

Área de estudo

O município de Monte Alegre está localizado na porção noroeste do estado do Pará, na mesorregião do Baixo Amazonas e Microrregião de Santarém, ocupando uma área aproximada de $20.400 \mathrm{~km}^{2}$ (Oliveira Júnior et al. 1999). A Serra do Itauajuri localiza-se no extremo sul deste município (Fig. 1) ocupando uma área de 1.598,21 hectares entre as coordenadas geográficas $1^{\circ} 50$ '3,55', $1^{\circ} 52^{\prime} 40,67^{\prime} ' \mathrm{~S}$ e $54^{\circ} 3^{\prime} 25,71^{\prime}$ ' $-54^{\circ} 9^{\prime} 50,6^{\prime \prime} \mathrm{W}$, com altitudes que variam de 40 a $440 \mathrm{~m}$, sendo considerada a maior serra nas proximidades do Rio Amazonas (Pará 2013).

Embora Monte Alegre apresente todas as formações vegetais que caracterizam o ambiente amazônico, na Serra do Itauajuri e em seu entorno predominam os campos naturais de terra firme formados por uma vegetação denominada Campinarana, caracterizada por uma associação entre elementos florísticos da mata das terras altas e espécies da flora dos campos ou campinas (Silva 2008). No topo da serra há uma mistura de fitofisionomias, que transitam desde formas campestres com afloramentos rochosos ou não, até formações florestais relativamente densas, como os cerradões e as matas de galeria (Pará 2013).

Além da variada fitofisionomia, a Serra do Itauajuri apresenta inúmeras cachoeiras e piscinas naturais de água cristalina, bem como nascentes, igarapés que podem secar no período de estiagem, ambientes cavernícolas e fauna diversificada (Pará 
2013). Geologicamente, a serra situa-se no flanco nordeste da estrutura denominada "Domo Monte Alegre", constituído por rochas sedimentares da Formação Faro, com idade estimada entre 325 e 345 milhões de anos (Pastana 1999).

A região está sob influência do clima tropical chuvoso tipo Am, segundo a classificação de Köppen, com média mensal de temperatura mínima superior a $18^{\circ} \mathrm{C}$, estação seca pouco duradoura, precipitações pluviométricas com cerca de 1.969 $\mathrm{mm}$, com distribuição irregular durante o ano, sendo dezembro a junho a época mais chuvosa, e julho a novembro a época mais seca, quando os índices totais mensais apresentam-se inferiores a 60 mm (Pará 2012).

\section{Amostragem e análise dos dados}

A coleta do material botânico foi realizada durante os meses de maio e julho de 2010 , em duas excursões, com duração de dez dias cada. As coletas ocorreram de forma aleatória através de busca ativa, visando contemplar os ambientes preferenciais à ocorrência de licófitas e samambaias, bem como o maior número possível de microhabitats (troncos de árvores, fendas nas rochas, barrancos úmidos, pedras úmidas, brejos, margens de cursos d'água). O material testemunho está depositado no herbário $\mathrm{MG}$, com duplicatas doadas ao BHCB e HBRA.

O sistema de classificação adotado para a linhagem das samambaias foi o proposto por Smith et al. (2006), já para a linhagem das licófitas (i.e. Selaginellaceae) seguiu-se Jermy (1990).

As formas de vida das espécies foram categorizadas de acordo com as definições de Lellinger (2002) e Zuquim et al. (2008) em: terrestres, rupícolas, epífitas, hemiepífitas, anfíbias e aquáticas flutuantes.
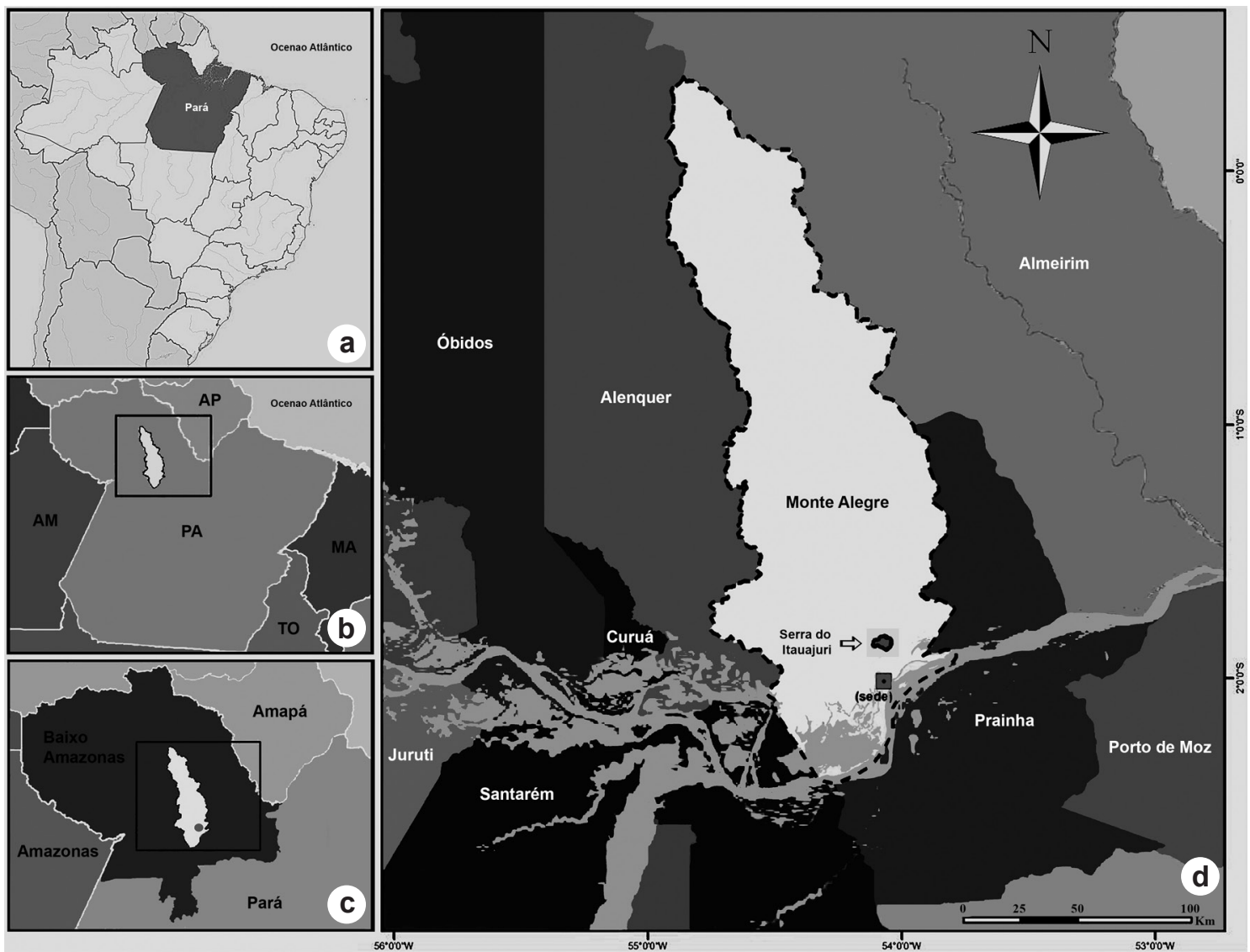

Figura 1 - Localização da área de estudo - a. estado do Pará, Brasil; b. município de Monte Alegre; c. localização de Monte Alegre na mesorregião do Baixo Amazonas; d. localização da Serra do Itauajuri no município de Monte Alegre. Figure 1 - Location of the studied area - a. Pará state, Brazil; b. municipality of Monte Alegre; c. location of Monte Alegre within the mesoregion of Baixo Amazonas; d. location of Serra do Itauajuri whitin the municipality of Monte Alegre. 
Para uma caracterização clara e objetiva das fitofisionomias presentes na área, foram estipulados critérios de acordo com a relação da vegetação e sua proximidade com cursos d'água. Desta forma, foram estabelecidos quatro ambientes de ocorrência para as espécies: mata ripária, sendo a formação florestal relacionada às áreas com menor altitude e geralmente em associação com os cursos d'água, podendo eventualmente ser alagada (Fig. 2e); mata de encosta, como sendo a formação florestal ocorrente na transição entre as áreas baixas e altas do local, não apresentando associação com
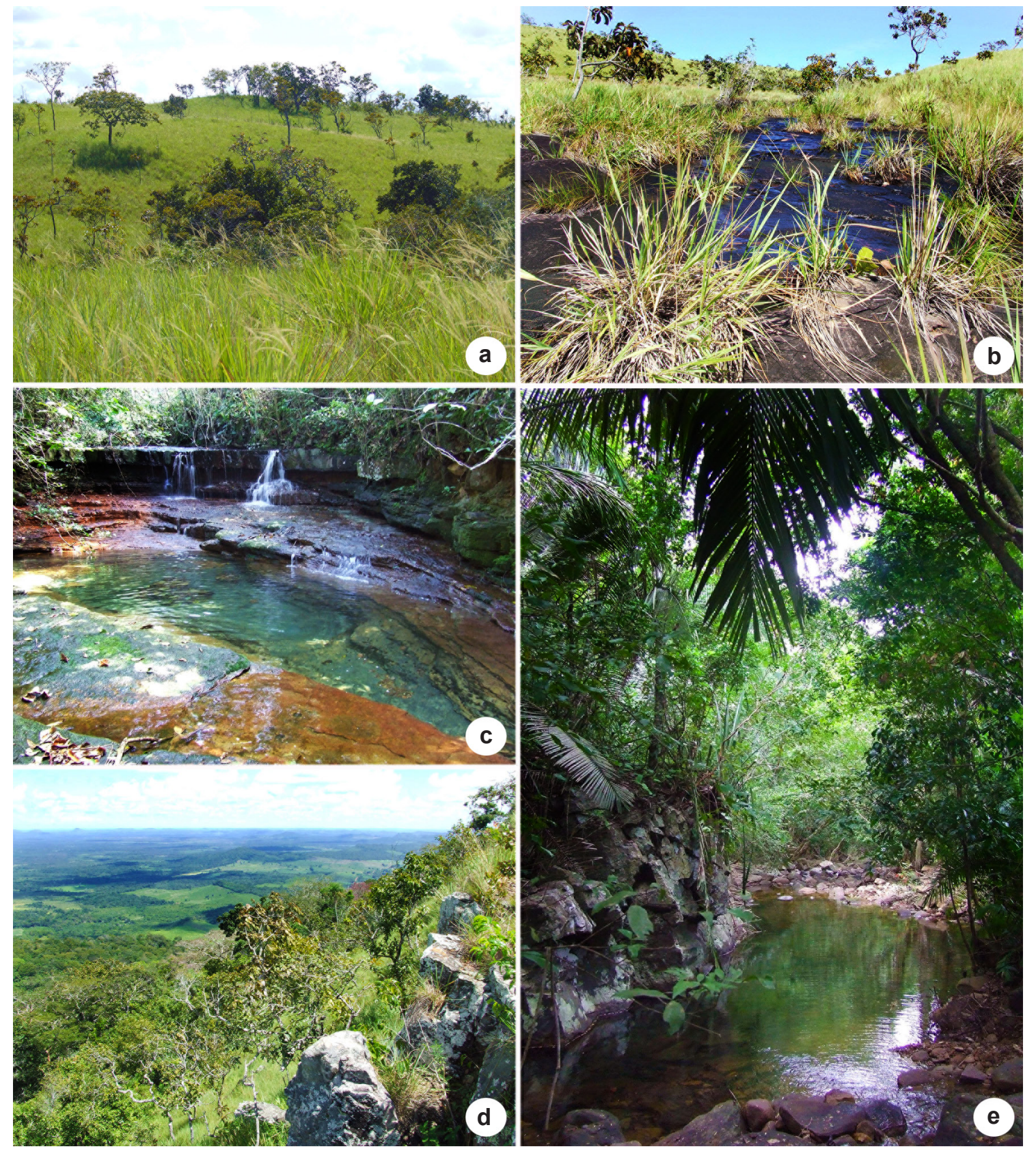

Figura 2 - Categorias de ambientes de ocorrência - a. formação campestre; b. formação campestre com afloramento rochoso; c. lagoa; d. mata de encosta; e. mata ripária.

Figure 2 - Categories of enviroment types - a. grassland vegetation; b. grassland vegetation with rocky outcrops; c. lagoon; d. hillside forest; e. riparian forest. 
cursos d'água (Fig. 2d); formação campestre, as formações abertas ocorrentes nas áreas mais altas, com vegetação herbáceo-arbustiva associada (Fig. 2ab); e lagoas, que embora não representem formações vegetais convencionais, são ambientes marcantes na área e, por isso, constituem aqui uma categoria independente (Fig. 2c).

A distribuição geográfica das espécies está de acordo com os padrões estabelecidos por Parris (2001), Moran \& Smith (2001) e Schwartsburd \& Labiak (2007), com as espécies podendo ser categorizadas em: circum-antárticas (presentes na América, África, Ásia e/ou Oceania), atlânticas (América, África e eventualmente ilhas do Oceano Atlântico), pacíficas (América, Ásia e/ou Oceania), americanas (Américas do Sul, Central e eventualmente do Norte), sul-americanas (apenas nos países da América do Sul) e endêmica do Brasil.

Para a análise das relações existentes entre as formas de vida e as fitofisionomias da área elaborou-se uma matriz de presença e ausência relacionando as formas de vida e a respectiva ocorrência nas fitofisionomias, seguindo Fraga \& Peixoto (2004). Posteriormente foram realizadas análises de similaridade utilizando-se o coeficiente de Sørenssen e algoritimo UPGMA (MuellerDumbois \& Ellemberg 1974), através do programa PAST (Hammer et al. 2001).

Assim como em Fraga \& Peixoto (2004), para ser possível visualizar quais as formas de vida que influenciam na associação das fitofisionomias, também foram analisadas a similaridade para cada forma de vida isolada, exceto para hemiepífitas, anfíbias e aquáticas flutuantes, já que estas apresentaram poucas espécies, sendo restritas a apenas uma fitofisionomia.

\section{Resultados e Discussão}

Foram registradas 64 espécies distribuídas em 36 gêneros e 21 famílias, sendo quatro espécies de licófitas e 60 de samambaias (Tab. 1). Em relação a outras áreas inventariadas no Pará, a Serra do Itauajuri apresenta uma riqueza de espécies relativamente elevada considerando sua pequena área (ca. 1.600 ha). O Parque Ambiental de Belém e a APA Ilha do Combu, ambos localizados na cidade de Belém, possuem aproximadamente a mesma área da Serra do Itauajuri, contudo, suas floras de licófitas e samambaias são compostas por apenas 49 e 26 espécies, respectivamente (Costa et al. 2006a; Ferreira et al. 2009). A Serra do Itauajuri, por outro lado, difere destas duas áreas por apresentar uma maior variedade de fitofisionomias, o que origina um maior número de microhabitats (Fig. 3), favorecendo a colonização por um maior número de espécies.

As famílias mais representativas foram Pteridaceae (19 spp.) e Polypodiaceae (8 spp.), as quais costumam estar dentre as de maior riqueza específica na Região (Costa et al. 2006a; Maciel et al. 2007; Costa \& Pietrobom 2007, 2010; Fernandes et al. 2012). No entanto, a representatividade específica de Hymenophyllaceae e Dryopteridaceae (2 spp. cada uma) contrasta com os resultados obtidos nos trabalhos supracitados, onde ambas as famílias destacaram-se pelo significativo número de espécies. Estas famílias geralmente estão representadas por espécies epífitas que ocorrem preferencialmente em ambientes úmidos no interior de matas fechadas, condições ambientais que não são predominantes na Serra do Itauajari.

Os gêneros com maior riqueza específica foram Adiantum L. (9 spp.) e Selaginella P. Beauv. (4 spp.), os quais, de acordo com Tryon \& Conant (1975), estão entre os mais representativos na Amazônia brasileira.

Em relação às formas de vida, foram registradas 35 espécies terrestres, 33 rupícolas e apenas 11 epífitas (Tab. 1). O significativo número de espécies rupícolas em detrimento das espécies epífitas difere do observado nas demais áreas inventariadas no Pará, onde existe ampla predominância de espécies terrestres e epífitas (Costa \& Pietrobom 2010). O resultado encontrado no presente estudo é influência direta das características ambientais da Serra, a qual possui diversos paredões e afloramentos rochosos, conferindo um cenário incomum ao que comumente é visto na Amazônia brasileira. Foram registradas ainda cinco espécies hemiepífitas, quatro anfíbias e quatro aquáticas flutuantes (Tab. 1).

Apesar da Serra do Itauajuri apresentar uma fitofisionomia variada com predomínio de formações campestres (Silva 2008; Pará 2013), 51 espécies (quase $80 \%$ ) ocorrem nas matas ripárias em áreas úmidas e sombreadas às margens de igarapés e interior da mata, sendo 38 espécies exclusivas deste ambiente (Tab. 1). Segundo Holttum (1938) e Santiago et al. (2004), a intensa luminosidade prejudica o estabelecimento de muitas espécies de plantas vasculares sem sementes, as quais tendem a se estabelecer em locais úmidos e sombreados. Esses locais oferecem condições favoráveis ao desenvolvimento destas plantas, em especial, nas fases iniciais de seu ciclo de vida, possibilitando sua sobrevivência. 
Nas matas de encosta, formações florestais não associadas a cursos d'água, foram registradas oito espécies, das quais apenas duas foram coletadas exclusivamente neste ambiente (Asplenium pumilum Sw. e Tectaria incisa Cav.). As lagoas de água cristalina, além de serem elementos marcantes na Serra do Itauajuri, também são locais de ocorrência de seis espécies de samambaias, com cinco destas crescendo como anfíbias ou aquáticas flutuantes apenas neste ambiente (Marsilea polycarpa Hook. \& Grev., Ceratopteris pteridoides (Hook.) Hieron., C. thalictroides (L.) Brongn.,
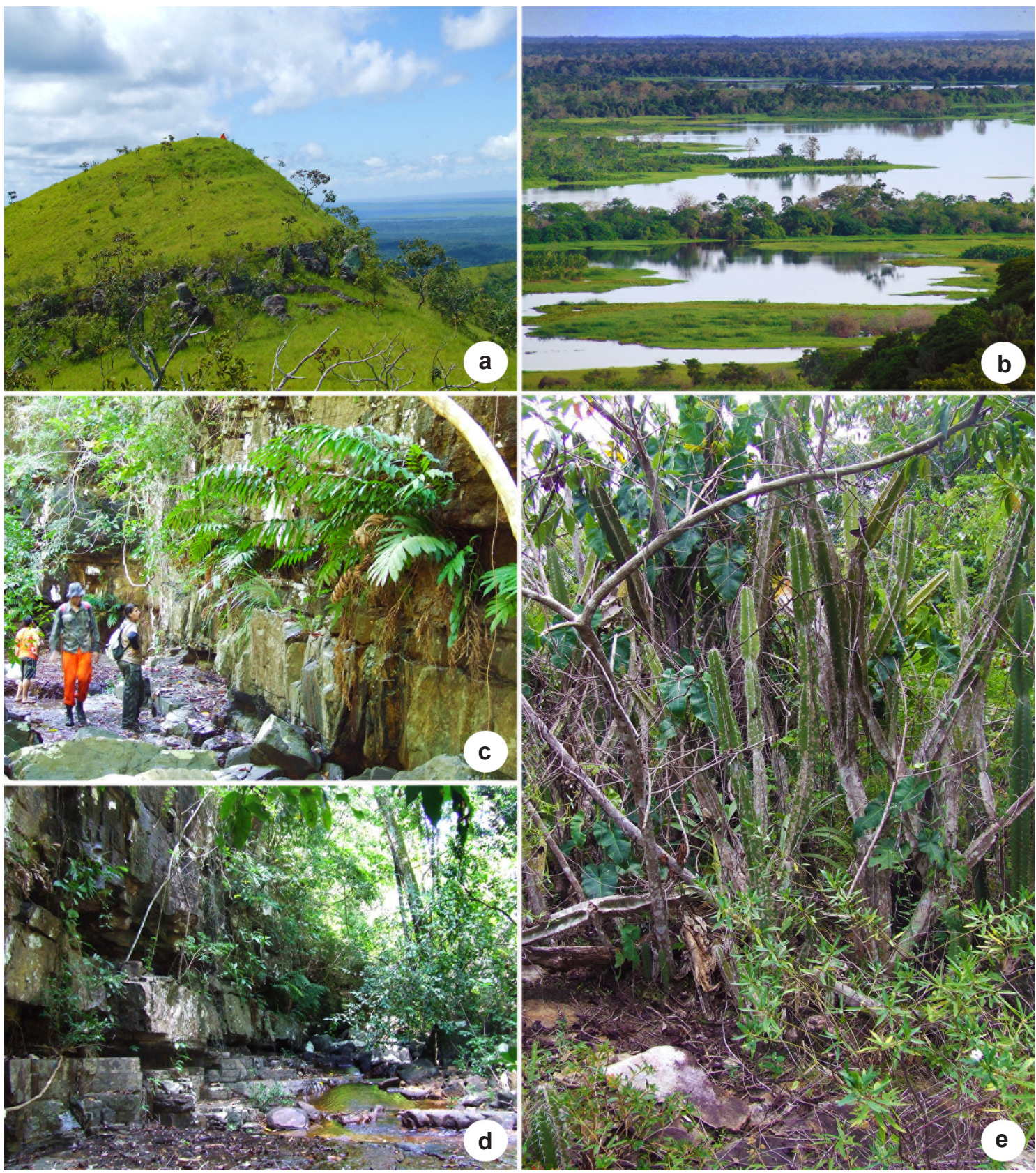

Figura 3 - Ambientes de ocorrência e microhabitats - a. topo da Serra do Itauajuri; b. mata ripária em áreas alagadas; c. paredão rochoso ao longo de um curso d'água; d. paredão rochoso em mata de galeria; e. ambiente xérico com espécies características. Figure 3 - Enviroment types and microhabitats - a. top of Serra do Itauajuri; b. riparian forest in flooded areas; c. rocky slope along stream; d. rocky slope in gallery forest; e. xeric environment with distinctive species. 
Tabela 1 - Formas de vida, ambientes de ocorrência e padrões de distribuição das licófitas e samambaias da Serra do Itauajuri. * Novos registros para a Região Norte. ** Novos registros para o estado do Pará. (AF: aquática flutuante; AN: anfíbia; EP: epífita; HE: hemiepífita; RU: rupícola; TE: terrestre. Ambientes de ocorrência - FC: formação campestre; LA: lagoa; ME: mata de encosta; MR: mata ripária).

Table 1 - Life-forms, environments types, and distribution patterns of lycophytes and ferns from Serra do Itauajuri. * New records to Northern Region. ** New records to Pará state. (AF: aquatic floating; AN: amphibious; EP: epiphyte; HE: hemiepiphyte; RU: rupicolous; TE: terrestrial. Enviroment types - FC: grassland vegetation; LA: lagoon; ME: hillside forest; MR: riparian forest).

\begin{tabular}{|c|c|c|c|c|}
\hline $\begin{array}{l}\text { LINHAGEM } \\
\text { Família } \\
\text { Espécie }\end{array}$ & $\begin{array}{l}\text { Formas de } \\
\text { vida }\end{array}$ & $\begin{array}{c}\text { Ambientes } \\
\text { de } \\
\text { ocorrência }\end{array}$ & $\begin{array}{c}\text { Padrão de } \\
\text { distribuição }\end{array}$ & Voucher \\
\hline
\end{tabular}

LICÓFITA

Selaginellaceae

Selaginella flagellata Spring

S. minima Spring **

S. sulcata (Desv. ex Poir.) Spring ex Mart.

S. tenella (P. Beauv.) Spring

SAMAMBAIA

Anemiaceae

Anemia buniiffolia (Gardner) T. Moore

A. oblongifolia (Cav.) Sw.

RU

RU

FC

Sul-Americana

Pallos \& Pietrobom 123

$\mathrm{MR}, \mathrm{FC}$

Americana

Pallos \& Pietrobom 154

Aspleniaceae

Asplenium cruegeri Hieron.

A. pumilum Sw. *

RU

MR

Americana

Pietrobom \& Ferreira 8572

A. serratum L.

RU, EP

ME

Atlântica

Pallos \& Pietrobom 122

Americana

Pallos \& Pietrobom 149

Blechnaceae

Blechnum heringeri Brade

B. polypodioides Raddi

B. serrulatum Rich.

Cyatheaceae

Cyathea microdonta (Desv.) Domin

Dennstaedtiaceae

Pteridium arachnoideum (Kaulf.) Maxon

Dryopteridaceae

Cyclodium meniscioides (Willd.) C. Presl var. meniscioides

Dryopteris huberi (Christ) C. Chr.
TE

FC

Americana

Pallos \& Pietrobom 128

TE, RU MR, FC

Endêmica do Brasil

TE

MR

Americana

Pallos \& Pietrobom 105

Pacífica

Pietrobom \& Ferreira 8548

TE

MR

Americana

Pietrobom \& Ferreira 8558

TE

MR

Sul-Americana

Pietrobom \& Ferreira 8546

RU

MR

Americana

Pallos \& Pietrobom 150
TE, RU MR 


\section{LINHAGEM \\ Família}

Espécie

Hymenophyllaceae

Trichomanes pedicellatum Desv.

T. pinnatum Hedw.

Lindsaeaceae

Lindsaea divaricata Klotzsch

L. pallida Klotzsch

L. stricta (Sw.) Dryand. var. stricta

Lomariopsidaceae

Nephrolepis biserrata (Sw.) Schott

N. brownii (Desv.) Hovenkamp \& Miyam.

Lygodiaceae

Lygodium venustum Sw.

L. volubile $\mathrm{Sw}$.

Marsileaceae

Marsilea ancylopoda A. Braun *

M. polycarpa Hook. \& Grev.

Metaxyaceae

Metaxya rostrata (Kunth) C. Presl.

Ophioglossaceae

Ophioglossum nudicaule L. f. **

Polypodiaceae

Campyloneurum phyllitidis (L.) C. Presl

Microgramma megalophylla (Desv.) de la Sota

M. percussa (Cav.) de la Sota

M. persicariifolia (Schrad.) C. Presl

Pecluma plumula (Humb. \& Bonpl. ex. Willd) M.G. Price

Phlebodium decumanum (Willd.) J. Sm.

Pleopeltis polypodioides (L.) E.G. Andrews \& Windham var. burchelli (Baker) A.R. Sm.

Serpocaulon triseriale (Sw.) A.R. Sm

Formas de
vida

Ambientes

de
Padrão de

distribuição ocorrência

Voucher

$\begin{array}{cccc}\text { HE } & \text { MR } & \text { Americana } & \text { Pietrobom \& Ferreira } 8547 \\ \text { TE, RU } & \text { MR } & \text { Americana } & \text { Pallos \& Pietrobom } 55\end{array}$

EP MR, FC Circum-Antártica Pallos \& Pietrobom 120

EP MR Pacífica Pietrobom \& Ferreira $8587 a$ $\begin{array}{cccc}\text { TE, RU } & \text { MR, ME, FC } & \text { Americana } & \text { Pallos \& Pietrobom } 36 \\ \text { TE } & \text { MR } & \text { Americana } & \text { Pietrobom \& Ferreira } 8587\end{array}$

$\begin{array}{cccc}\text { AN } & \text { MR } & \text { Americana } & \text { Pietrobom \& Ferreira } 8550 \\ \text { AF } & \text { LA } & \text { Pacífica } & \text { Pietrobom \& Ferreira } 8542\end{array}$

RU MR Americana Pallos \& Pietrobom 65

TE FC Circum-Antártica Pietrobom \& Ferreira 8538

RU MR Americana Pallos \& Pietrobom 151

RU, EP MR, ME Sul-Americana Pallos \& Pietrobom 66

$\begin{array}{cccc}\text { RU } & \text { MR } & \text { Americana } & \text { Pietrobom \& Ferreira } 8562 \\ \text { RU, EP } & \text { MR } & \text { Americana } & \text { Pallos \& Pietrobom } 147 \\ \text { EP } & \text { MR } & \text { Americana } & \text { Pallos \& Pietrobom } 84\end{array}$

TE, RU, EP MR, ME, FC Americana Pallos \& Pietrobom 79

RU, EP MR Sul-Americana Pallos \& Pietrobom 40

RU MR Americana Pallos \& Pietrobom 127 


\begin{tabular}{lcccc}
\hline LINHAGEM & Formas de & Ambientes & Padrão de & Voucher \\
Família & vida & ocorrência & distribuição & Espécie \\
\hline
\end{tabular}

Psilotaceae

Psilotum nudum (L.) P. Beauv.

EP

Pteridaceae

Adiantopsis radiata (L.) Fée

A. senae (Baker) Schuettp. \& Al. Davila

Adiantum deflectens Mart.

A. humile Kunze

A. latifolium Lam.

A. lunulatum Burm. Fl.

A. obliquum Willd.

A. petiolatum Desv.

A. pulverulentum $\mathrm{L}$.

A. terminatum Kunze ex Miq.

A. tetraphyllum Humb. \& Bonpl. ex Willd.

Ananthacorus angustifolius (Sw.) Underw. \& Maxon

Ceratopteris pteridoides (Hook.) Hieron.

C. thalictroides (L.) Brongn.

Hemionitis palmata L.

H. rufa (L.) Sw.

Pityrogramma calomelanos (L.) Link var. calomelanos

Vittaria lineata (L.) Sm.

Salviniaceae

Azolla filiculoides Lam.

Salvinia auriculata Aubl.

Schizaeaceae

Schizaea elegans (Vahl) Sw.

Tectariaceae

Tectaria incisa Cav.

Triplophyllum funestum (Kunze) Holttum

$\mathrm{AF}$

AF

TE
Pteris tripartita Sw.

$\begin{array}{cccc}\text { TE } & \text { MR } & \text { Americana } & \text { Pallos \& Pietrobom } 107 \\ \text { RU } & \text { FC } & \text { Sul-Americana } & \text { Pallos \& Pietrobom } 125 \\ \text { TE, RU } & \text { MR } & \text { Americana } & \text { Pallos \& Pietrobom } 50 \\ \text { TE } & \text { MR } & \text { Americana } & \text { Pallos \& Pietrobom } 109 \\ \text { TE } & \text { MR } & \text { Americana } & \text { Pallos \& Pietrobom } 47 \\ \text { TE } & \text { MR } & \text { Circum-Antártica } & \text { Pietrobom \& Ferreira } 8579 \\ \text { TE } & \text { MR } & \text { Americana } & \text { Pallos \& Pietrobom } 133 \\ \text { TE, RU } & \text { MR, ME } & \text { Americana } & \text { Pallos \& Pietrobom } 83 \\ \text { TE, RU, EP } & \text { MR, ME } & \text { Americana } & \text { Pallos \& Pietrobom } 102 \\ \text { TE, RU } & \text { MR } & \text { Americana } & \text { Pallos \& Pietrobom } 164 \\ \text { TE } & \text { MR } & \text { Americana } & \text { Pallos \& Pietrobom } 129 \\ \text { RU } & \text { ME, FC } & \text { Americana } & \text { Pallos \& Pietrobom } 89 \\ & & & \\ \text { AF } & \text { LA } & \text { Americana } & \text { Pietrobom \& Ferreira } 8540 \\ \text { AN } & \text { LA } & \text { Circum-Antártica } & \text { Pietrobom \& Ferreira } 8554 \\ \text { TE, RU } & \text { MR } & \text { Americana } & \text { Pallos \& Pietrobom } 54 \\ \text { TE, RU } & \text { MR } & \text { Americana } & \text { Pallos \& Pietrobom } 33 \\ \text { TE, RU, AN } & \text { MR, LA } & \text { Americana } & \text { Pallos \& Pietrobom } 115 \\ \text { TE } & \text { MR } & \text { Circum-Antártica } & \text { Pietrobom \& Ferreira } 8551 \\ & \text { MR } & \text { Americana } & \text { Pallos \& Pietrobom } 46 \\ & & & \end{array}$

LA Americana

Pietrobom \& Ferreira 8555

LA

Americana

Pietrobom \& Ferreira 8557

MR

Americana

Pallos \& Pietrobom 158

$\begin{array}{llll}\text { TE } & \text { ME } & \text { Americana } & \text { Pallos \& Pietrobom 130 } \\ \text { TE } & \text { MR } & \text { Americana } & \text { Pallos \& Pietrobom 141 }\end{array}$

Thelypteridaceae

Thelypteris interrupta (Wiild.) K. Iwats. TE, AN MR Circum-Antártica

Pietrobom \& Ferreira 8549

T. longifolia (Desv.) R.M. Tryon
MR
Americana 
Azolla filiculoides Lam. e Salvinia auriculata Aubl.). Nas formações campestres, fitofisionomia predominante na área, foram registradas 15 espécies, das quais cinco foram observadas exclusivamente nesta formação (Anemia buniifolia (Gardner) T. Moore, Pteridium arachnoideum (Kaulf.) Maxon, Ophioglossum nudicaule L.f., Psilotum nudum (L.) P. Beauv. e Adiantopsis senae (Baker) Schuettp. \& Al. Davila) (Tab. 1).

Analisando o dendrograma do Coeficiente de Sørenssen para as espécies ocorrentes em cada fitofisionomia da área (Fig. 4a) percebe-se uma maior relação entre as formações campestres e a
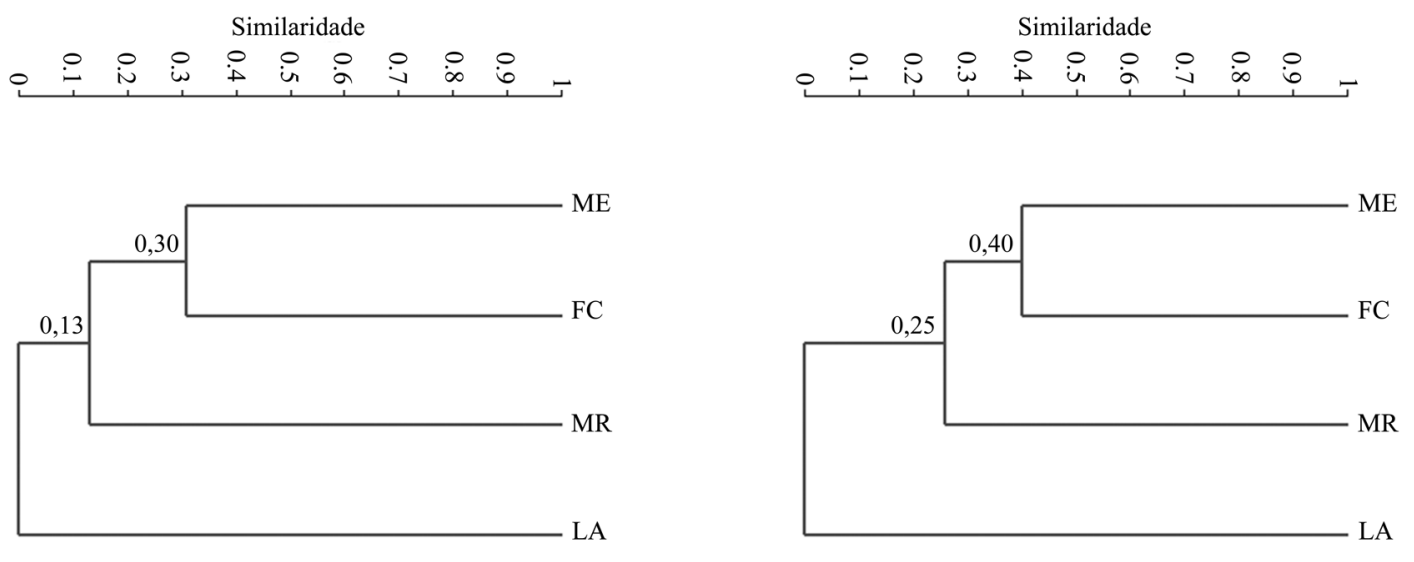

a

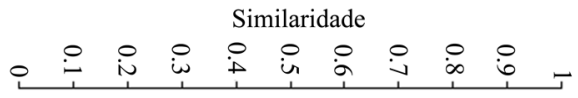

mata de encosta (índice de 0,3), estando estas duas relacionadas às matas ripárias (índice de 0,13 ). $\mathrm{O}$ ambiente de lagoa, como esperado, possui baixíssima relação com as demais fitofisionomias analisadas (índice de 0,001), já que neste ambiente ocorrem espécies praticamente exclusivas (Tab. 1). Tal relação mostra uma clara associação das fitofísionomias com o incremento de altitude e a disponibilidade de água em cada ambiente. Essa associação influencia a ocorrência das formas de vida de cada espécie em cada fitofisionomia, já que é de se esperar características similares em ambientes semelhantes (Halloy \& Mark 1996; Kluge \& Kessler 2007).
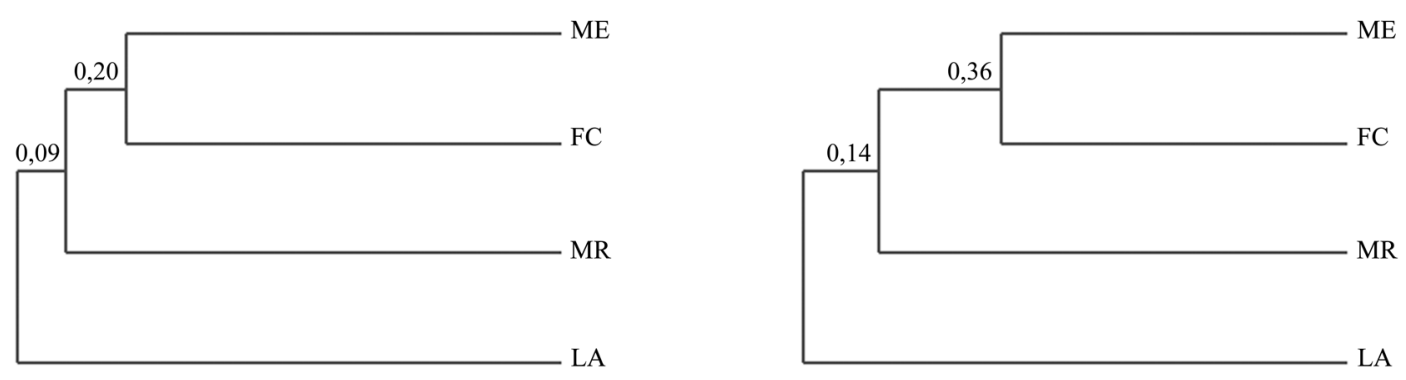

C

Figura 4 - Dendrogramas de similaridade florística de Sørenssen entre as fitofisionomias - a. todas as espécies (coeficiente cofenético: 0,96); b. espécies epífitas (coeficiente cofenético: 0,96); c. espécies rupícolas (coeficiente cofenético: 0,82); d. espécies terrestres (coeficiente cofenético: 0,99). FC: formação campestre; LA: lagoa; ME: mata de encosta; MR: mata ripária. Figure 4 - Florisric similarity dendrogram using Sørenssen coefficient of fitofisionomies - a. all species (cophenetic coefficient: 0,96); b. epiphitic species (cophenetic coefficient: 0,96); c. epipetric species (cophenetic coefficient: 0,82); d. terrestrial species (cophenetic coefficient: 0,99). FC: grassland vegetation; LA: lagoon; ME: hillside forest; MR: riparian forest. 
Para verificar se as formas de vida encontradas são influenciadas pelas fitofisionomias, comparouse o dendrograma originado pela totalidade das espécies (Fig. 4a) com os dendrogramas de cada forma de vida separadamente (Figs. 4b-d). Desta forma percebeu-se que tanto para as espécies epífitas (Fig. 4b), quanto para as rupícolas (Fig. 4c) e as terrestres (Fig. 4d) as relações encontradas são mantidas, apenas apresentando valores diferentes nos agrupamentos. Isso provavelmente ocorre pelo elevado número de espécies com formas de vida exclusivas de cada ambiente na Serra do Itauajuri, o que evidencia que as formas de vida são influenciadas por cada uma das fitofisionomias da área. Por exemplo, Adiantum petiolatum Desv. e Blechnum heringeri Brade apresentam formas de vida diferentes em cada fitofisionomia que ocorrem (Tab. 1).

Em relação ao padrão de distribuição geográfica, foram registradas 46 espécies americanas (ca. 70\%), sete circum-antárticas, seis sul-americanas, três pacíficas e uma atlântica (Tab. 1). Não foram registradas espécies endêmicas da Amazônia brasileira, corroborando as informações trazidas por Tryon \& Conant (1975) sobre a biogeografia deste grupo, que é o endemismo muito baixo ou ausente nesta região. Foi registrada uma espécie endêmica do Brasil (Blechnum heringeri), porém, sua distribuição ultrapassa os limites amazônicos, ocorrendo também em áreas de cerrado em Goiás, no Distrito Federal e em Minas Gerais (Prado et al. 2015).

No que diz respeito ao estado de conservação das espécies inventariadas, duas delas encontramse na Lista Vermelha do Centro Nacional de Conservação da Flora (CNCFlora). A espécie Blechnum heringeri encontra-se incluída na categoria "Vulnerável" (CNCFlora 2015), sendo considerada rara e pouco conhecida, com pouquíssimos registros em herbários (V.A.O. Dittrich, comunicação pessoal). Além disso, seu registro na Serra do Itauajuri representa o limite mais setentrional de sua distribuição. Já a espécie Schizaea elegans (Vahl) Sw. encontra-se incluída na categoria "Menos Preocupante" (CNCFlora 2015), possuindo registros no Acre, Amazonas, Pará, Rondônia, Roraima, Tocantins, Alagoas, Pernambuco, Bahia, Mato Grosso e em todos os estados das regiões Sul e Sudeste do Brasil (Prado et al. 2015 ). De acordo com Zuquim et al. (2008) esta é uma espécie relativamente rara, que cresce em ambientes variados, desde áreas úmidas e sombreadas até áreas abertas e secas, onde formam adensamentos. Ainda segundo estes autores, S. elegans pode representar, na verdade, um complexo com mais de uma espécie.

Como em estudos anteriores (i.e., Costa et al. 2006a-b; Costa \& Pietrobom 2007; Maciel et al. 2007; Costa \& Pietrobom 2010; Fernandes et al. 2012; Góes-Neto \& Pietrobom 2012; Góes-Neto et al. 2015), onde são apresentados novos registros para o estado do Pará, para a Região Norte do Brasil (Amazônia brasileira) e até mesmo para o Brasil, no presente estudo também são citados dois novos registros para a Região Norte do país (Asplenium pumilum Sw. e Marsilea ancylopoda A. Braun) e dois para o Pará (Ophioglossum nudicaule L. e Selaginella minima Spring). Estes resultados em particular reafirmam a importância dos estudos florísticos para o conhecimento da flora de uma região, especialmente na Amazônia brasileira onde há inúmeras lacunas de coleta.

Diferentemente do que se observa nas extensas florestas de planície da Amazônia, a Serra do Itauajari possui características ambientais bastante distintas, apresentando inúmeros paredões e afloramentos rochosos, com matas ripárias e de encosta, em transição para formações campestres. Sendo assim, além de apresentar uma flora de licófitas e samambaias rica e abundante, a referida Serra apresenta elementos florísticos diferenciados, uma vez que predominam as espécies rupícolas sobre as epífitas, fato nunca antes registrado nos trabalhos realizados no Pará.

Portanto, a Serra do Itauajari é uma área biologicamente importante, cuja riqueza da flora de licófitas e samambaias, bem como de outros grupos de seres vivos, só poderá ser conservada com o adequado manejo e preservação das formações vegetais da área. Neste sentido, os resultados obtidos neste trabalho reúnem informações importantes que podem, juntamente com o Relatório Técnico da Secretaria de estado de Meio Ambiente do Pará, subsidiar a criação e a implementação da Unidade de Conservação da Serra do Itauajuri, o que garantirá a preservação de suas riquezas históricas e naturais.

\section{Agradecimentos}

À Coordenação de Aperfeiçoamento de Pessoal de Nível Superior (CAPES), a bolsa de Mestrado concedida à primeira autora e de Doutorado concedida ao segundo autor. Ao Incra - Oeste do Pará, o transporte durante as excursões de coleta e a facilitação do acesso à área estudada. 
À família Ferreira, a estadia oferecida e o apoio logístico durante as excursões de coleta. Ao Sr. Roberto de Jesus, o auxílio em campo. Ao Dr. Vinícius Dittrich, os comentários sobre a espécie Blechnum heringeri. Agradecemos também aos dois revisores anônimos que trouxeram importantes contribuições a este trabalho. Este artigo é parte da dissertação de Mestrado da primeira autora.

\section{Referências}

Avila-Pires, T.C.S.; Hoogmoed, M.S. \& Rocha, W.A. 2010. Notes on the vertebrates of northern Pará, Brazil: a forgotten part of the Guianan Region, I. Herpetofauna. Boletim do Museu Paraense Emílio Goeldi - Ciências Naturais 5: 13-112.

Brasil. 2008. Plano Amazônia Sustentável: Diretrizes para o desenvolvimento sustentável da Amazônia brasileira. Ministério do Meio Ambiente, Brasília. $112 \mathrm{p}$.

CNCFlora. 2015. Centro Nacional de Conservação da Flora. Lista vermelha. Disponível em $<$ http:// cncflora.jbrj.gov.br/portal/pt-br/listavermelha $>$. Acesso em 28 julho 2015.

Costa, J.M. \& Pietrobom, M.R. 2007. Pteridófitas (Lycophyta e Monilophyta) da Ilha de Mosqueiro, município de Belém, estado do Pará, Brasil. Boletim do Museu Paraense Emílio Goeldi Ciências Naturais 2: 45-55.

Costa, J.M. \& Pietrobom, M.R. 2010. Samambaias e licófitas do Parque Estadual do Gunma, município de Santa Bárbara do Pará, estado do Pará, Brasil. Rodriguésia 61: 223-232.

Costa, J.M.; Souza, M.G.C. \& Pietrobom, M.R. 2006a. Levantamento florístico das pteridófitas (Lycophyta e Monilophyta) do Parque Ambiental de Belém (Belém, Pará, Brasil). Revista de Biologia Neotropical 3: 4-12.

Costa, J.M.; Pietrobom, M.R. \& Souza, M.G.C. 2006 b. Primeiro registro de Trichomanes pinnatinervium Jenman (Hymenophyllaceae - Monilophyta) para o Brasil. Bradea 11: 33-36.

Fernandes, R.S.; Maciel, S. \& Pietrobom, M.R. 2012. Licófitas e monilófitas das Unidades de Conservação da Usina Hidroelétrica - UHE de Tucuruí, Pará, Brasil. Hoehnea 39: 247-285.

Ferreira, L.S.L.; Costa, J.M. \& Pietrobom, M.R. 2009. As pteridófitas. In: Jardim, M.A.G. (org.). Diversidade biológica das áreas de proteção ambiental Ilhas do Combu e Algodoal-Maiandeua, Pará, Brasil. MPEG/MCT/CNPq, Belém. Pp. 13-40.

Fraga, C.N. \& Peixoto, A.L. 2004. Florística e ecologia das Orchidaceae das restingas do estado do Espírito Santo. Rodriguésia 55: 5-20.

Góes-Neto, L.A.A. \& Pietrobom, M.R. 2012. Novos registros de samambaias para a Amazônia Brasileira. Rodriguésia 63: 1151-1155.
Góes-Neto, L.A.A.; Maciel, S.; Pietrobom, M.R. \& Valdespino, I.A. 2015. Licófitas (Lycopodiophyta) do Corredor de Biodiversidade do Norte do Pará, Brasil. Rodriguésia 66: 229-244.

Halloy, S.R.P. \& Mark, A.F. 1996. Comparative leaf morphology spectra of plant communities in New Zealand, the Andes and the European Alps. Journal of The Royal Society of New Zealand 26: 41-78.

Hammer, Ø.; Harper, D.A.T. \& Ryan, P.D. 2001. PAST: Paleontological statistics software package for education and data analysis. Paleontologia Electronica 4: 1-9.

Holttum, R.E. 1938. The ecology of tropical pteridophytes. In: Verdoom, F.R. (ed.). Manual of Pteridology. Martinus Nijhoff, The Hague. Pp. 420-450.

Jermy, A.C. 1990. Selaginellaceae. In: Kramer, K.U. \& Green, P.S. (eds.). Pteridophytes and gymnosperms Vol. I. In: Kubitzki, K. (ed.). The families and genera of vascular plants. Springer-Verlag, Berlin. Pp. 39-45.

Kluge, J. \& Kessler, M. 2007. Morphological characteristics of fern assemblages along an elevational gradient: patterns and causes. Ecotropica 13: 27-43.

Lellinger, D.B. 2002. A modern multilingual glossary for taxonomic pteridology. American ferns Society, Inc, Washington, DC. 263p.

Maciel, S.; Souza, M.G. \& Pietrobom, M.R. 2007. Licófitas e monilófitas do Bosque Rodrigues Alves Jardim Botânico da Amazônia, município de Belém, estado do Pará, Brasil. Boletim do Museu Paraense Emílio Goeldi - Ciências Naturais 2: 1-27.

Moran, R.C. \& Smith, A.R. 2001. Phytogeographic relationships between neotropical and AfricanMadagascar pteridophytes. Brittonia 53: 304-351.

Mueller-Dombois, D. \& Ellenberg, H. 1974. Aims and methods of vegetation ecology. J. Wiley \& Sons Press, New York. 547p.

Oliveira Júnior, R.C.; Rodrigues, T.E.; Santos, P.L. \& Valente, M.A. 1999. Zoneamento Agroecológico do município de Monte Alegre, estado do Pará. Embrapa Amazônia Oriental, Belém. 87p.

Pará. 2012. Estatística Municipal - Monte Alegre. Governo do estado do Pará. Secretaria de Estado de Planejamento, Orçamento e Finanças. Disponível em <http://seicom.pa.gov.br/kitmineracao/ estatistica-municipal/regiao-do-baixo-amazonas/ MonteAlegre.pdf $>$. Acesso em 28 julho 2015.

Pará. 2013. Relatório técnico para a criação de unidade de conservação (município de Monte Alegre). Secretaria Especial de Estado de Infraestrutura e Logística para o Desenvolvimento Sustentável/ Secretaria de Estado de Meio Ambiente. Disponível em <http://www.sema.pa.gov.br/wp-content/ uploads/2014/10/Relatorio_Tecnico_Criacao UC_Serra_do_Itauajuri_22102014.pdf $>$. Acesso em 28 julho 2015. 
Parris, B.S. 2001. Circum-Antarctic continental distribution patterns in pteridophyte species. Brittonia 53: 270-283.

Pastana, J.M.N. 1999. Síntese geológica e favorabilidades para tipos de jazimentos minerais: município de Monte Alegre. Programa informações para gestão territorial. Estado do Pará. CPRM, Monte Alegre. $42 \mathrm{p}$.

Prado, J. \& Moran, R.C. 2009. Checklist of the ferns and lycophytes of Acre state, Brazil. Fern Gazette 18: 230-263.

Prado, J.; Sylvestre, L.S.; Labiak, P.H.; Windisch, P.G.; Salino, A.; Barros, I.C.L.; Hirai, R.Y.; Almeida, T.E.; Santiago, A.C.P.; Kieling-Rubio, M.A.; Pereira, A.F.N.; Øllgaard, B.; Ramos, C.G.V.; Mickel, J.T.; Dittrich, V.A.O.; Mynssen, C.M.; Schwartsburd, P.B.; Condack, J.P.S.; Pereira, J.B.S. \& Matos, F.B. 2015. Diversity of ferns and lycophytes in Brazil. Rodriguésia 66: 1073-1083.

Ricci, M. 1996. Variation in distribution and abundance of the endemic flora of Juan Fernández Island, Chile. Pteridophyta. Biodiversity and Conservation 5: $1521-1532$.

Santiago, A.C.P.; Barros, I.C.L. \& Sylvestre, L.S. 2004. Pteridófitas ocorrentes em três fragmentos florestais de um brejo de altitude (Bonito, Pernambuco, Brasil). Acta Botanica Brasílica 18: 781-792.
Schwartsburd, P.B. \& Labiak, P.H. 2007. Pteridófitas do Parque Estadual de Vila Velha, Ponta Grossa, Paraná, Brasil. Hoehnea 34: 159-209.

Silva, J.M.C.; Rylands, A.B. \& Fonseca, G.A.B. 2005. The fate of the Amazonian areas of endemism. Conservation Biology 19: 689-694.

Silva, M.R.P. \& Rosário, S.M. 2008. Licófitas e monilófitas (Pteridophyta) da Floresta Nacional de Caxiuanã, estado do Pará, Brasil: chave para as famílias e as espécies de Aspleniaceae e Blechnaceae. Boletim do Museu Paraense Emílio Goeldi - Ciências Naturais 3: 151-163.

Silva, R.O. 2008. Biodiversidade e políticas de conservação: o caso do Parque Estadual Monte Alegre - Pará. Tese de Doutorado. Universidade de Brasília, Brasília. 300p.

Smith, A.R.; Pryer, K.M.; Schuettpelz, E.; Korall, P.; Schneider, H. \& Wolf, P.G. 2006. A classification for extant ferns. Taxon 55: 705-731.

Tryon, R.M. \& Conant, D.S. 1975. The ferns of Brazilian Amazonia. Acta Amazonica 5: 23-24.

Tryon, R.M. \& Tryon, A.F. 1982. Ferns and allied plants, with special reference to Tropical America. SpringerVerlag, New York, Heidelberg, Berlin. 857p.

Zuquim, G.; Costa, F.R.C.; Prado, J. \& Tuomisto, H. 2008. Guia de samambaias e licófitas da REBIO Uatumã, Amazônia Central. INPA, Manaus. 316p. 